\title{
Ferramentas do coaching no desenvolvimento da liderança da enfermagem na atenção primária à saúde*
}

Coaching tools in the development of nursing leadership in primary health care

Herramientas de coaching para desenvolvimiento de lideranza de enfermeria en atención primaria de salud

\section{Bárbara Souza Rocha', Denize Bouttelet MunariI", Bruna Mendes de Sousa ${ }^{\mathrm{III}}$, Maria Lúcia Leal ${ }^{\mathrm{IV}}$, Luana Cássia Miranda Ribeirov}

Resumo: Objetivo: descrever o uso de ferramentas do coaching no processo de desenvolvimento da liderança de enfermeiros que atuam na Atenção Primária à Saúde (APS). Método: estudo descritivo, de abordagem qualitativa, do tipo pesquisa-ação, em que os dados da aplicação das ferramentas Roda da Vida e Meta E.S.P.E.R.T.A para nove enfermeiros foram organizados no software WebQDA e analisados por categorização de conteúdo. Resultados: os participantes vivenciaram três ações principais: autoconhecimento, estabelecimento de metas e consolidação de mudanças. As mudanças aconteceram ao delegar responsabilidades, no equilíbrio emocional, ao lidar com conflitos e na comunicação. Considerações finais: as ferramentas favoreceram a identificação de potencialidades e limitações e direcionaram o processo de mudança atitudinal e comportamental pelo estabelecimento de metas. O estudo apresentou característica inovadora, unindo experimentação e objetivação, levando os enfermeiros à incorporação de mudanças no cotidiano do trabalho e na liderança da APS.

Descritores: Enfermagem de Atenção Primária; Liderança; Inovação Organizacional

Abstract: Objective: To describe the use of coaching tools in the leadership development process of nurses working in Primary Health Care (PHC). Method: A descriptive study with a qualitative approach, of an action research type, in which data from the application of the Wheel of Life and E.S.P.E.R.T.A

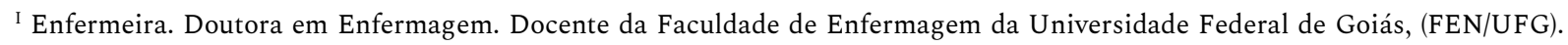
Goiânia, Goiás. Brasil; E-mail: barbarasrocha@gmail.com; ORCID ID: https://orcid.org/0000-0001-6059-8399

${ }^{\text {II }}$ Enfermeira. Doutora em Enfermagem. Docente Aposentada da Faculdade de Enfermagem da Universidade Federal de Goiás, (FEN/UFG). Goiânia, Goiás. Brasil. E-mail: boutteletmunari@gmail.com; ORCID ID: https://orcid.org/0000-0002-2225-770X

III Graduanda em Enfermagem pela Universidade Federal de Goiás, (FEN/UFG). Goiânia, Goiás. Brasil. E-mail: bmds.enfermagem@gmail.com; ORCID ID: https://orcid.org/0000-0003-3793-2031

IV Psicóloga. Mestre em Psicologia Social pela Pontifícia Universidade Católica de Goiás. Goiânia, Goiás. Brasil. E-mail: lucia.sobrap@gmail.com ORCID ID: https://orcid.org/0000-0001-8003-9172

${ }^{\vee}$ Enfermeira. Doutora em Enfermagem. Docente da Faculdade de Enfermagem da Universidade Federal de Goiás, (FEN/UFG). Goiânia, Goiás. Brasil. E-mail: luaufg@yahoo.com.br ORCID ID: https://orcid.org/0000-0002-4254-2030

*Artigo oriundo da tese de doutorado intitulada: DESENVOLVIMENTO DE LIDERANÇA PARA ENFERMEIROS DA SAÚDE DA FAMÍlIA COM O USO DA ESTRATÉGIA COACHING EM GRUPO defendida no Programa de Pós-Graduação em Enfermagem da Faculdade de Enfermagem da Universidade Federal de Goiás, em 02 de Dezembro de 2014
} 
Ferramentas do coaching no desenvolvimento da liderança da enfermagem na atenção... I 2

Goal tools for nine nurses were organized in WebQDA software and analyzed by content categorization. Results: The participants experienced three main actions: self-knowledge, goal setting and change consolidation. The changes happened after delegating responsibilities, in the emotional balance, when dealing with conflict, and in communication. Final considerations: the tools favored the identification of potentialities and limitations and directed the process of attitudinal and behavioral change by setting goals. The study presented an innovative characteristic, combining experimentation and objectification, leading nurses to incorporate changes in daily work and PHC leadership. Keywords: Primary Care Nursing; Leadership; Organizational Innovation

Resumen: Objetivo: describir el uso de herramientas de coaching en proceso desenvolvimiento de lideranza de enfermeros que actuan en Atención Primaria a la Salud (APS). Método: estúdio descriptivo, abordaje cualitativa, tipo investigación-acción, en datos de aplicación herramientas Rueda de Vida y Meta E.S.P.E.R.T.A, nueve enfermeros fueron organizados en software WebQDA y analizados por categorias de contenido. Resultados: los participantes vivenciaron tres acciones principales: autoconocimiento, establecimiento de metas y consolidación de cambios. Los cambios pasaron a delegar responsabilidades, en equilíbrio emocional, lidar con conflictos y en la comunicación. Consideraciones finales: las herramientas favorecen en identificación de potencialidad, limitaciones y direccionan el processo de cambios actitudinales de comportamiento por establecimiento de metas. El estúdio presento característica inovadora, uniendo experimentación y objetivación, llevando los enfermeros a la incorporación de cambios en su cotidiano de trabajo y lideranza de APS.

Descriptores: Enfermería de Atención Primaria, Liderazgo, Innovación Organizacional

\section{Introdução}

Enfermeiros têm assumido cada vez mais papéis de liderança ao longo de toda a cadeia de prestação de cuidados em saúde. Suas responsabilidades vão desde o processo de cuidado até a tomada de decisões estratégicas no ambiente executivo das organizações. ${ }^{1-2}$

No contexto da Atenção Primária à Saúde (APS), o enfermeiro é referência na articulação das ações gerenciais e de cuidado, planejando, executando, monitorando e avaliando as ações da equipe e dos profissionais sob sua supervisão. ${ }^{3-4}$ Por esta razão, o processo de formação dos enfermeiros para a liderança é importante, ${ }^{5}$ especialmente na APS, onde se requerem, desses profissionais, competências gerenciais para garantir a continuidade da estratégia de Saúde da Família $(\mathrm{SF})^{4}$ e o fortalecimento dos princípios da APS. ${ }^{4,6-7}$ 
Tais competências possibilitam uma atuação proativa do enfermeiro no sistema de saúde como um todo, tendo "maior envolvimento no diálogo político e tomada de decisão relativa ao planejamento, desenvolvimento e avaliação de serviços e políticas”.7:5 A formação de enfermeiros em liderança deve ser parte de iniciativas institucionais e de políticas públicas de saúde, visando a um alvo muito além da simples necessidade individual dos profissionais em se aperfeiçoar nessa área. ${ }^{2-5}$

Estudos ${ }^{2,5,8}$ têm sinalizado a importância de programas de desenvolvimento de lideranças. Entre as estratégias metodológicas desses programas, o coaching tem sido apontado como estratégia potente tanto no desenvolvimento da capacidade de liderar como na produção de mudanças importantes no comportamento de líderes de alto desempenho. ${ }^{9-10} \mathrm{O}$ coaching é um processo que favorece o autoconhecimento e o estabelecimento de metas por meio de uma análise profunda do indivíduo, provocando uma reflexão sobre perspectivas, mentalidades, crenças e abordagens que podem levar a um comportamento mais sustentável, favorecendo a capacidade de autoliderança. Em suma, o coaching é um processo que promove o indivíduo de um estado atual para um estado desejado. ${ }^{11-12}$

Na literatura em Enfermagem, é evidente o impacto do uso das ferramentas do coaching no desenvolvimento de competências gerenciais e na formação de novos enfermeiros gestores., ${ }^{2,-10,12-16}$ No entanto, a maior parte dos estudos se restringe à formação de enfermeiros na área hospitalar ${ }^{2,10,12-15}$ ou ainda no campo do ensino. ${ }^{16}$

Em uma busca realizada em bases de dados nacionais e internacionais, não se encontrou aplicação do coaching na APS, em particular, relacionada ao desenvolvimento de lideranças em Enfermagem nesse contexto. Estudos ${ }^{9,15}$ salientam a carência de pesquisas na Enfermagem utilizando ferramentas do coaching, o que 
Ferramentas do coaching no desenvolvimento da liderança da enfermagem na atenção... I 4

aponta uma importante lacuna. Assim sendo, como as ferramentas do coaching auxiliam no desenvolvimento da liderança de enfermeiros que atuam na APS?

Com base nessa justificativa é que se propôs este estudo, cujo objetivo foi descrever o uso de ferramentas do coaching no processo de desenvolvimento da liderança de enfermeiros que atuam na APS.

A investigação traz uma contribuição inovadora por mostrar o potencial do coaching na produção de mudanças no desempenho de enfermeiros e de sua importância estratégica na capacitação de enfermeiros da APS.

\section{Método}

Estudo descritivo, de abordagem qualitativa, fundamentada na pesquisa-ação (PA), considerado um tipo de pesquisa-intervenção que pressupõe o envolvimento do pesquisador e do participante em uma relação, de caráter colaborativo e emancipatório, com o intuito de promover mudanças para melhorar a prática. ${ }^{17} \mathrm{~A}$ escolha da metodologia fundamentou-se na sua adequação para sustentar a proposta da pesquisa, simultaneamente articulada a um programa de desenvolvimento de liderança $(\mathrm{DL})$ para enfermeiros.

O programa de DL (quadro 1) aconteceu durante sete meses, tempo em que foram realizados sete encontros em grupo, com duração média de seis horas cada, totalizando 42 horas de intervenção. Os encontros aconteciam a cada vinte dias, todos às quartas-feiras, sempre na mesma sala. Além disso, os enfermeiros tiveram uma assessoria individual de quatro horas. Após seis meses do término do programa, foi realizado um grupo focal de avaliação.

\begin{tabular}{|c|c|c|c|c|}
\hline $\begin{array}{c}\mathbf{N}^{\circ} \text { do } \\
\text { encontro }\end{array}$ & Tema & $\begin{array}{c}\mathbf{N}^{\text {o de }} \\
\text { participantes }\end{array}$ & Objetivo & $\begin{array}{c}\text { Mês do } \\
\text { encontro }\end{array}$ \\
\hline $1^{\circ}$ & $\begin{array}{c}\text { Diagnóstico e } \\
\text { contrato de } \\
\text { trabalho com o }\end{array}$ & 21 & $\begin{array}{c}\text { Identificar a situação atual dos } \\
\text { enfermeiros na liderança; } \\
\text { Elaborar o contrato do grupo; }\end{array}$ & Jun/2012 \\
\hline
\end{tabular}




\begin{tabular}{|c|c|c|c|c|}
\hline & grupo. & & Assinar TCLEs. & \\
\hline $2^{\circ}$ & $\begin{array}{l}\text { Nucleação do } \\
\text { Grupo. }\end{array}$ & 19 & $\begin{array}{l}\text { Vivenciar a nucleação do grupo ao } \\
\text { autoconhecimento; } \\
\text { Elaborar a "Roda da Vida". }\end{array}$ & Ago/2012 \\
\hline $3^{\circ}$ & $\begin{array}{l}\text { Comunicação e } \\
\text { Feedeback. }\end{array}$ & 15 & $\begin{array}{l}\text { Diagnosticar a comunicação no } \\
\text { grupo, na vida e no trabalho; } \\
\text { Compreender a teoria do feedback. }\end{array}$ & Set/2012 \\
\hline $4^{\circ}$ & $\begin{array}{l}\text { Papel do } \\
\text { enfermeiro na } \\
\text { liderança. }\end{array}$ & 12 & $\begin{array}{c}\text { Definir os papéis do enfermeiro na } \\
\text { liderança. }\end{array}$ & Out/2012 \\
\hline $5^{\circ}$ & Liderança. & 12 & $\begin{array}{c}\text { Entender o conceito aprofundado de } \\
\text { liderança. }\end{array}$ & Nov/2012 \\
\hline $6^{\circ}$ & $\begin{array}{l}\text { Planejamento e } \\
\text { Mudança. }\end{array}$ & 15 & $\begin{array}{l}\text { Criar planejamento estratégico - } \\
\text { método da árvore; } \\
\text { Elaborar a meta individual. }\end{array}$ & Nov/2012 \\
\hline $\begin{array}{l}\text { Assessoria } \\
\text { Individual }\end{array}$ & $\begin{array}{l}\text { META } \\
\text { E.S.P.E.R.T.A. }\end{array}$ & 17 & $\begin{array}{c}\text { Revisar a meta e definir passos em } \\
\text { direção à mudança. }\end{array}$ & Dez/2012 \\
\hline $7^{\circ}$ & $\begin{array}{l}\text { Necessidades e } \\
\text { Líder Coach. }\end{array}$ & 16 & $\begin{array}{c}\text { Identificar as necessidades de } \\
\text { mudança e compreender o papel do } \\
\text { líder coach. }\end{array}$ & Dez/2012 \\
\hline
\end{tabular}

Quadro 1: Apresentação do programa de DL, Goiânia, 2014

Esse programa de DL foi fundamentado no coaching ${ }^{11}$ por acreditar nas possibilidades deste na potencialização de mudanças na atuação dos enfermeiros no contexto da APS. O coaching, predominantemente, é um processo realizado em formato individual, mas pode ser empregado no formato grupal quando um grupo de indivíduos, com suas questões atuais, se reúne para trabalhar em parceria com um facilitador para desenvolver, em grupo, planos para solucionar as dificuldades apontadas. $^{11}$

Para o desenvolvimento da investigação, utilizaram-se as recomendações do Consolidated Criteria for Reporting Qualitative Research (COREQ). ${ }^{18}$ A pesquisa foi conduzida pela pesquisadora principal, que tem formação básica em dinâmica de grupo e é coach certificada por instituição internacional. ${ }^{11}$ Além disso, contou com uma equipe constituída por uma observadora, também com formação básica em dinâmica de grupo, duas psicólogas organizacionais do Instituto Brasileiro de 
Psicanálise, Dinâmica de Grupo e Psicodrama (SOBRAP/GOIÁS) e com a supervisão da orientadora, todas com formação em coaching. ${ }^{11}$

A investigação foi realizada junto à Secretaria Municipal de Saúde (SMS) de uma capital do Centro-Oeste brasileiro com enfermeiros da Saúde da Família (SF). Foi realizada uma reunião inicial de mobilização onde foram esclarecidos os detalhes e as condições para a participação no programa de DL e sua vinculação com a pesquisa. Os enfermeiros deveriam ter graduação em Enfermagem, atuação mínima de um ano na SF, vínculo efetivo com a SMS, interesse e disponibilidade em participar e, ainda, concordar com a metodologia adotada.

Foram convidados a participar do estudo 24 enfermeiros. No primeiro encontro, compareceram 21. Foram convidados novamente, para o segundo encontro, os 24 enfermeiros. No segundo encontro, compareceram 19 enfermeiros. A partir daí, aqueles enfermeiros que não participaram de nenhum encontro foram excluídos do estudo. O número de participantes por encontro está descrito no Quadro 1, apresentado anteriormente.

No primeiro encontro do programa de DL, após esclarecimentos sobre a pesquisa, foi solicitada a assinatura do Termo de Consentimento Livre e Esclarecido (TCLE) aos interessados e foram estabelecidas, em conjunto, as regras de convivência e participação.

A coleta de dados ocorreu entre os meses de fevereiro de 2012 a junho de 2013, período em que aconteceu o programa de DL (sete encontros e a assessoria individual) e o grupo focal de avaliação, onde foi possível identificar as mudanças decorrentes desse processo. Os dados foram extraídos do registro nas ferramentas do coaching utilizadas e por meio de gravação em áudio das discussões em grupo. Para este estudo, 
7 I Rocha BS, Munari DB, Sousa BM, Leal ML, Ribeiro LCM

em particular, foram pesquisadas as ferramentas do coaching: Roda da Vida e a Meta E.S.P.E.R.T.A. ${ }^{11}$

A Roda da Vida (RV) teve sua origem nos anos 60 e foi atribuída a Paul J. Meyer. É uma ferramenta simples de autoconhecimento, pois, basicamente, é um círculo dividido em áreas da vida que são consideradas importantes para o sucesso. Neste estudo, a RV original foi adaptada e aplicada no segundo encontro com o grupo. Cada participante preencheu a RV considerando-se a escala de zero a $100 \%$ de satisfação em relação às 12 áreas previamente definidas pelos pesquisadores, que tratavam de aspectos da vida pessoal e/ou profissional. A pergunta central a ser respondida no preenchimento da RV foi: “Como cheguei aqui?”.

As partes da roda referiam-se a: 1. Administração do tempo; 2. Capacidade de dar e receber feedback; 3. Competência gerencial; 4. Comunicação (saber ouvir e saber expressar); 5. Equilíbrio da vida pessoal; 6. Inteligência emocional; 7. Lidar com conflitos; 8. Liderança; 9. Relacionamento interpessoal; 10. Saúde e boa forma; 11 e 12. Áreas opcionais (lazer, capacidade de lidar com pressão, desenvolvimento profissional, organização pessoal, finanças e espiritualidade). Os participantes poderiam escolher dentre essas opções.

Já a Meta E.S.P.E.R.T.A (ME) foi aplicada no sexto encontro, quando o grupo começou o processo de definição de metas individuais para melhorar o exercício da liderança. Foi revisada em sessão individual e/ou quando solicitada pelo participante mesmo fora dos horários previstos. O formulário da ME favorece a definição de metas por meio do preenchimento de seus componentes nas iniciais da palavra "esperta" e seus significados, correspondendo, respectivamente, a: específica; sistêmica; passos; evidências; recursos; tesão; alternativas/adversidades. Os participantes elaboraram 
suas metas de mudanças a partir da questão: "Analisando minha roda da vida, o que eu quero mudar?”.

Para armazenamento e organização dos dados de registro das ferramentas do coaching, foi utilizado o software WebQDA. Já na análise e interpretação dos resultados, utilizou-se o método de Análise de Conteúdo, ${ }^{19}$ em que o processo de categorização dos dados se deu no sistema por “acervo", quando a classificação analógica e progressiva dos dados gerou as categorias ${ }^{19}$.

Este estudo respeitou todos os preceitos éticos de pesquisa com seres humanos e foi aprovado pelo Comitê de Ética em Pesquisa do Hospital das Clínicas/UFG sob o protocolo $\mathrm{N}^{\circ} .171 / 2011$, com prorrogação de prazo autorizado pelo mesmo comitê de ética no dia 03 de dezembro de 2015, atendendo à legislação vigente constante na Resolução 466/2012. No sentido de preservar a identidade dos participantes, seus registros são apresentados pela letra “E” seguida de números (de um a nove).

\section{Resultados}

Os resultados apresentados no estudo referem-se a nove participantes do programa de DL que preencheram as duas ferramentas utilizadas. Destes, seis eram supervisores no âmbito distrital e três, enfermeiros que integravam a equipe local.

A primeira categoria, chamada de Autoconhecimento, reflete o diagnóstico situacional individual dos enfermeiros em relação ao exercício da liderança na SF, de acordo com o registro da Roda da Vida.

Na sequência, ressaltou-se o estabelecimento de metas, a partir do uso da Meta E.S.P.E.R.T.A, no qual foi demonstrado o processo de criação de metas de mudança a serem alcançadas no programa de DL. Finalmente, a categoria Consolidação de mudanças apresenta uma avaliação geral do processo vivenciado pelos enfermeiros, 
reforçando as mudanças e apresentando os efeitos produzidos a partir do programa de DL.

\section{Autoconhecimento}

Ao elaborarem sua RV individual, os enfermeiros demonstraram que, em algumas áreas, seu grau de satisfação era inferior a 50\%, como a inteligência emocional, a administração do tempo, a capacidade de lidar com pressão e a capacidade de dar e receber feedback. Apontam-se esses dados, uma vez que estes influenciaram diretamente a criação das metas que mobilizaram os enfermeiros no processo de mudança. No gráfico 1, mostra-se a porcentagem de enfermeiros que atribuíram nota inferior a 50\% de satisfação a determinadas áreas.

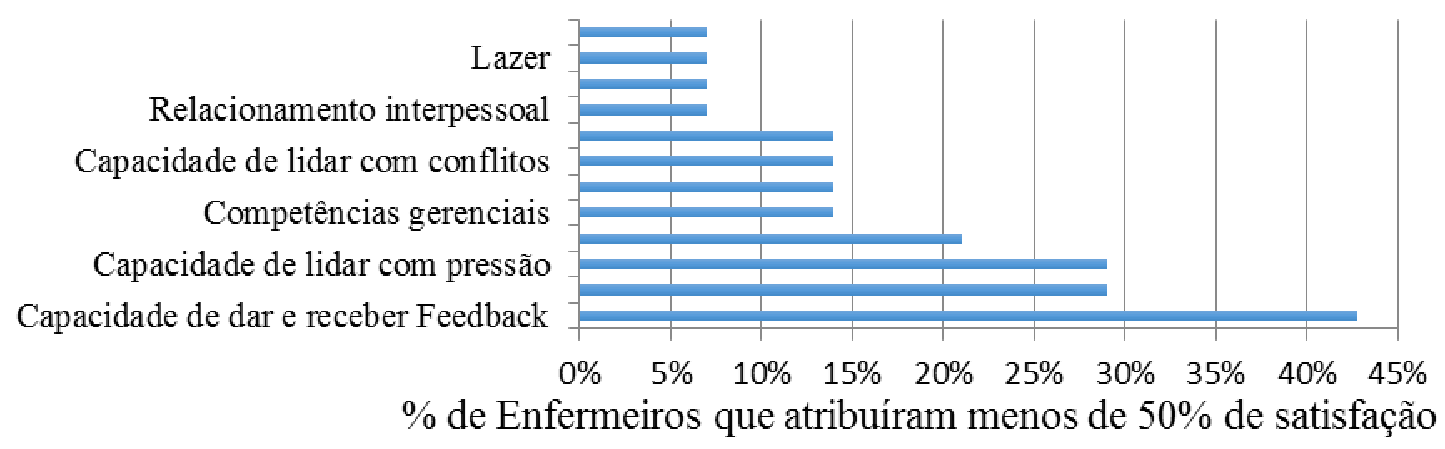

Gráfico 1 - Distribuição das áreas que receberam, por parte dos enfermeiros, grau de satisfação inferior a 50\% (GOIÂNIA, 2014).

\section{Estabelecimento de metas}

O uso da Roda da Vida favoreceu a tomada de consciência, por parte dos enfermeiros, sobre as áreas de maior fragilidade. Esse movimento orientou a identificação dos aspectos prioritários que necessitavam de maior atenção e desenvolvimento. A partir desse momento, iniciou-se a priorização das mudanças almejadas pelos enfermeiros e o uso da ME facilitou a organização do pensamento. O 
Ferramentas do coaching no desenvolvimento da liderança da enfermagem na atenção... | 10

quadro 2 ilustra as metas definidas pelos enfermeiros e a descrição de cada um dos seus componentes.

Rev. Enferm. UFSM, Santa Maria, v. 9, p. 1-23, 2019 


\begin{tabular}{|c|c|c|c|c|c|c|c|}
\hline & $\begin{array}{l}\text { ESPECÍFICA: O que você } \\
\text { quer especificamente? }\end{array}$ & $\begin{array}{l}\text { SISTÊMICA: Como } \\
\text { esta meta vai afetar } \\
\text { rocê e os outros? }\end{array}$ & $\begin{array}{l}\text { PASSOS: Quais são os passos } \\
\text { hecessários? }\end{array}$ & $\begin{array}{l}\text { EVIDÊNCIAS: } \\
\text { Eomo saberá que } \\
\text { stá conseguindo? }\end{array}$ & $\begin{array}{l}\text { RECURSOS: Quais } \\
\text { ecursos pessoais } \\
\text { rai mobilizar? }\end{array}$ & $\begin{array}{l}\text { TESÃO: Qual a } \\
\text { mportância desta } \\
\text { neta para a sua } \\
\text { ida? }\end{array}$ & $\begin{array}{l}\text { ADVERSIDADES: } \mathrm{O} \\
\text { que o poderia impedir? }\end{array}$ \\
\hline E1) & $\begin{array}{l}\text { idar efetivamente com os } \\
\text { fonflitos e dizer não de } \\
\text { orma assertiva. }\end{array}$ & $\begin{array}{l}\text { Criar um clima mais } \\
\text { harmônico na } \\
\text { equipe, tornando-a } \\
\text { mais coesa, produtiva } \\
\text { tranquila; preservar } \\
\text { abertura para } \\
\text { idarem com o } \\
\text { problema; ter boa } \\
\text { elação com a equipe. }\end{array}$ & $\begin{array}{l}\text { Adquirir conhecimento; } \\
\text { puvir com imparcialidade; } \\
\text { star disponível, ser parte da } \\
\text { equipe; terapia, falar não } \\
\text { dentro do contexto. }\end{array}$ & $\begin{array}{l}\text { piminuir } \quad \text { os } \\
\text { fonflitos; registro } \\
\text { do desenvolvimento } \\
\text { de como falar não. }\end{array}$ & $\begin{array}{l}\text { Pabedoria; bom } \\
\text { penso; manejo; } \\
\text { permitir-se; } \\
\text { ppoiar-se nas } \\
\text { egras e nos } \\
\text { fonhecimentos; } \\
\text { poio do diretor. }\end{array}$ & $\begin{array}{l}\text { Piminuir demandas } \\
\text { conflitos; } \\
\text { fumprimento das } \\
\text { hormas } \\
\text { nstitucionais. }\end{array}$ & $\begin{array}{l}\text { Medo de perder as } \\
\text { poas relações, medo da } \\
\text { ná resposta; dar um } \\
\text { empo para dar a } \\
\text { esposta definitiva; } \\
\text { espirar e dar um } \\
\text { empo. }\end{array}$ \\
\hline E2) & $\begin{array}{l}\text { Paber delegar; ter clareza na } \\
\text { minha fala (objetiva/direta); } \\
\text { irmeza nas minhas ideias e } \\
\text { ppiniões; dar e receber } \\
\text { feedback. }\end{array}$ & $\begin{array}{l}\text { Ser respeitada, me } \\
\text { sentir mais realizada; } \\
\text { nais segurança ao } \\
\text { grupo. }\end{array}$ & $\begin{array}{l}\text { Reconhecer e priorizar o que } \\
\text { importante para mim; } \\
\text { puscar uma forma de falar } \\
\text { mais objetiva. }\end{array}$ & $\begin{array}{lr}\text { Analisar } & 0 \\
\text { esultado } & \text { das } \\
\text { pituações; registrar } \\
\text { atos. }\end{array}$ & Plan & & $\begin{array}{l}\text { Falta de educação das } \\
\text { pessoas. }\end{array}$ \\
\hline E3) & $\begin{array}{l}\text { fontrolar melhor minhas } \\
\text { emoções; manter a calma ao } \\
\text { alar em público; falar } \\
\text { pausadamente; lidar melhor } \\
\text { fom situações de estresse. }\end{array}$ & $\begin{array}{l}\text { Ser melhor } \\
\text { fompreendida; } \\
\text { diminuição do } \\
\text { sofrimento pelas } \\
\text { ooisas do trabalho. }\end{array}$ & 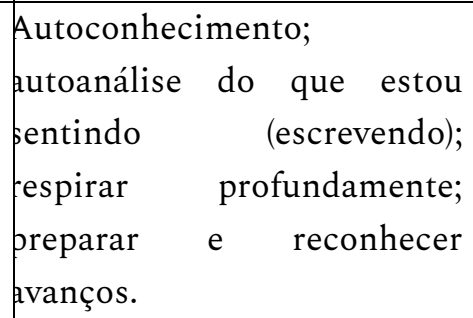 & $\begin{array}{l}\text { fomportar-me de } \\
\text { orma calma nas } \\
\text { pituações de } \\
\text { pressão; as pessoas } \\
\text { ão notar. }\end{array}$ & $\begin{array}{l}\text { Investir em } \\
\text { écnicas de falar } \\
\text { em público. }\end{array}$ & $\begin{array}{l}\text { Maturidade; } \\
\text { fonseguir me } \\
\text { expressar melhor; } \\
\text { per melhor } \\
\text { fompreendida. }\end{array}$ & $\begin{array}{l}\text { Situações de estresse, } \\
\text { pressão, hormônios. }\end{array}$ \\
\hline E4) & $\begin{array}{l}\text { Priorizar as tarefas a mim } \\
\text { stabelecidas. }\end{array}$ & $\begin{array}{lr}\text { Haver } & \text { melhor } \\
\text { distribuição } & \text { das } \\
\text { ninhas } & \text { tarefas; devo } \\
\text { sempre } & \text { lembrar o } \\
\text { quanto } & \text { posso }\end{array}$ & $\begin{array}{l}\text { Par prioridade às minhas } \\
\text { arefas, mas também pedir e } \\
\text { fferecer ajuda. }\end{array}$ & $\begin{array}{l}\text { Repassando o que é } \\
\text { do outro para o } \\
\text { putro e esperar o } \\
\text { seu tempo. }\end{array}$ & $\begin{array}{l}\text { Paciência, } \\
\text { olerância } \\
\text { aprender } \\
\text { fonhecer o tempo } \\
\text { do outro. }\end{array}$ & $\begin{array}{lr}\text { Ganharei } & \text { mais } \\
\text { ranquilidade } & \mathrm{e} \\
\text { onforto. } & \end{array}$ & $\begin{array}{l}\text { nvadir o espaço do } \\
\text { putro. }\end{array}$ \\
\hline
\end{tabular}

Rev. Enferm. UFSM, Santa Maria, v. 9, p. 1-19, 2019 
Ferramentas do coaching no desenvolvimento da liderança da enfermagem na atenção... I 12

\begin{tabular}{|c|c|c|c|c|c|c|c|}
\hline & & $\begin{array}{l}\text { Eolaborar com } 0 \\
\text { putro. }\end{array}$ & & & & & \\
\hline E5) & $\begin{array}{l}\text { pentir-me menos cansada; } \\
\text { perceber que o outro está me } \\
\text { alorizando. }\end{array}$ & $\begin{array}{l}\text { Ter boa relação } \\
\text { nterpessoal e menos } \\
\text { gressividade. }\end{array}$ & $\begin{array}{l}\text { Respirar para organizar meus } \\
\text { pensamentos; explicar como } \\
\text { me sinto e buscar evidências; } \\
\text { ão demorar a dar respostas } \\
\text { conversar sobre o fato). }\end{array}$ & $\begin{array}{l}\text { Estourar menos; } \\
\text { nenos ansiedade; } \\
\text { er mais feliz } \\
\text { fomigo mesma. }\end{array}$ & $\begin{array}{l}\text { Terapia; trabalhar } \\
\text { mais } \\
\text { mutoestima minha } \\
\text { erapia; foco para } \\
\text { tingir minha } \\
\text { meta. }\end{array}$ & $\begin{array}{lr}\text { fuidar da } & \text { saúde; } \\
\text { diminuir } & \text { estresse } \\
\text { has } & \text { relações } \\
\text { nterpessoais } & \text { e } \\
\text { fetivas. } & \end{array}$ & $\begin{array}{l}\text { Aumento dos } \\
\text { feedbacks negativos, } \\
\text { paixa autoestima. }\end{array}$ \\
\hline E6) & $\begin{array}{l}\text { dentificar, com a equipe, as } \\
\text { dificuldades; analisar o que } \\
\text { e pode construir juntos para } \\
\text { nelhorar a nossa } \\
\text { omunicação } \\
\text { ansequentemente, nossas } \\
\text { ações. }\end{array}$ & $\begin{array}{l}\text { Trabalhar mais o } \\
\text { puvir, o observar. }\end{array}$ & $\begin{array}{l}\text { Agendar e realizar reuniões } \\
\text { oletivas e individuais; } \\
\text { pprender a fazer perguntas } \\
\text { obre as consequências do } \\
\text { que faz. }\end{array}$ & $\begin{array}{ll}\text { fapacidade } & \text { de } \\
\text { puvir } & \text { mais } \\
\text { lesenvolvida; } & \text { mais } \\
\text { olerância. } & \end{array}$ & $\begin{array}{l}\text { Parceria com } \\
\text { putros } \\
\text { profissionais. }\end{array}$ & $\begin{array}{l}\text { Melhorar minha } \\
\text { qualidade de vida. }\end{array}$ & $\begin{array}{l}\text { Exercício } \\
\text { eutopercepção. }\end{array}$ \\
\hline E7) & $\begin{array}{l}\text { Pefinir planejamento e } \\
\text { rrioridades de forma } \\
\text { onsensual com a equipe em } \\
\text { nédio prazo. }\end{array}$ & $\begin{array}{lr}\text { Melhorar } & \text { o } \\
\text { elacionamento na } & \text { na } \\
\text { equipe; perceber } \\
\text { rodutividade } & \text { da } \\
\text { rópria equipe; ter } \\
\text { naior clareza na } \\
\text { fomunicação. }\end{array}$ & $\begin{array}{l}\text { Fazer cronograma diário, } \\
\text { emanal e checklist das } \\
\text { tividades diárias; } \\
\text { ensibilizar a equipe; visitar } \\
\text { eções e projeto; priorizar a } \\
\text { eitura sobre planejamento. }\end{array}$ & $\begin{array}{l}\text { Mais satisfação da } \\
\text { quipe; cronograma } \\
\text { por escrito; } \\
\text { lanejamento } \\
\text { pendo executado; } \\
\text { melhor } \\
\text { pomunicação. }\end{array}$ & $\begin{array}{l}\text { Buscar } \\
\text { onhecimentos } \\
\text { pobre } \\
\text { planejamento. }\end{array}$ & $\begin{array}{l}\text { Realização } \\
\text { profissional, } \\
\text { ntegração } \\
\text { equipe } \\
\text { pupervisão } \\
\text { omunicação } \\
\text { fetiva. }\end{array}$ & $\begin{array}{l}\text { alta de foco; falta de } \\
\text { disciplina; } \\
\text { ntercorrências. }\end{array}$ \\
\hline (E8) & $\begin{array}{l}\text { Eu quero fazer o que é meu e } \\
\text { hão o do todo quando eu } \\
\text { onseguir diminuir as } \\
\text { pbrigações e fazer algumas } \\
\text { que gosto, mas não faço } \\
\text { omo lazer. }\end{array}$ & $\begin{array}{l}\text { lou me sentir } \\
\text { nelhor ao ajudar as } \\
\text { pessoas; continuar } \\
\text { om espírito de ajuda } \\
\text { aprender a dar e } \\
\text { eceber feedback. }\end{array}$ & $\begin{array}{l}\text { Pizer não assertivo e } \\
\text { prientado; saber fortalecer os } \\
\text { putros, ajudando-os nas } \\
\text { arefas, acompanhando e } \\
\text { obrando; criar estratégia } \\
\text { para aumentar a tolerância; } \\
\text { brir espaço para lazer. }\end{array}$ & $\begin{array}{l}\text { Processo de } \\
\text { autoavaliação } \\
\text { mplementado; } \\
\text { erei mais tempo } \\
\text { para o lazer. }\end{array}$ & $\begin{array}{lr}\text { Mais } & \text { tolerância; } \\
\text { ncentivar } & \text { maior } \\
\text { nteresse } & \text { e } \\
\text { notivação } & \text { das } \\
\text { pessoas; } & \text { aceitar } \\
\text { ijuda; } & \text { perguntar } \\
\text { mais } & \text { sobre }\end{array}$ & $\begin{array}{l}\text { é uma questão } \\
\text { essoal de } \\
\text { prendizado; acho } \\
\text { que sentirei melhor } \\
\text { terei tempo para } \\
\text { dministrar minha } \\
\text { ida pessoal e }\end{array}$ & $\begin{array}{l}\text { Mau hábito das } \\
\text { pessoas em me } \\
\text { procurarem toda hora. }\end{array}$ \\
\hline
\end{tabular}

Rev. Enferm. UFSM, Santa Maria, v. 9, p. 1-23, 2019 


\begin{tabular}{|c|c|c|c|c|c|c|c|}
\hline & & & & & $\begin{array}{l}\text { hjuda/solução que } \\
\text { ps colegas podem } \\
\text { ne dar. }\end{array}$ & $\begin{array}{l}\text { puscar } \quad \text { outros } \\
\text { prazeres. }\end{array}$ & \\
\hline E9) & $\begin{array}{l}\text { Ter coragem de colocar a } \\
\text { ninha opinião sem me } \\
\text { preocupar com a reação do } \\
\text { putro; aprender a me } \\
\text { falorizar; reconhecer êxito; } \\
\text { dentificar qualidades. }\end{array}$ & $\begin{array}{l}\text { Per corajosa e } \\
\text { arriscar mais; sentir- } \\
\text { ne mais realizada; } \\
\text { nelhorar o humor - } \\
\text { ps outros poderão } \\
\text { discordar de mim; } \\
\text { preservar } \\
\text { ponderação; } \\
\text { fompartilhar elogios. }\end{array}$ & $\begin{array}{l}\text { Registrar minhas qualidades } \\
\text { avaliá-las; processo de } \\
\text { cceitação; listar êxitos; } \\
\text { xercício diário de } \\
\text { omemorar e validação. }\end{array}$ & $\begin{array}{l}\text { Vou me expor mais, } \\
\text { pem medo de não } \\
\text { per aceita; falar com } \\
\text { mais assertividade } \\
\text { coragem. }\end{array}$ & $\begin{array}{l}\text { niciativa própria; } \\
\text { nnálise diária das } \\
\text { ninhas ações: } \\
\text { oram como devem } \\
\text { per? }\end{array}$ & $\begin{array}{l}\text { Essa meta me ajuda } \\
\text { a ter mais segurança } \\
\text { a reconhecer o } \\
\text { neu valor enquanto } \\
\text { profissional. }\end{array}$ & $\begin{array}{l}\text { Alguém me criticar e } \\
\text { me desanimar. }\end{array}$ \\
\hline
\end{tabular}

Quadro 2 - Descrição das Metas ESPERTA estabelecidas pelos enfermeiros. Goiânia, 2014

\section{Consolidação de mudanças}

A consolidação das mudanças planejadas por meio do uso das ferramentas do coaching foi identificada durante o grupo focal realizado após seis meses de encerramento do programa. Nesse encontro, os enfermeiros avaliaram os resultados do programa de DL, especialmente, revisitando a sua RV e indicando modificações no nível de satisfação das áreas mais frágeis. O quadro 3 ilustra a comparação de alguns aspectos pontuados pelos enfermeiros considerados frágeis na elaboração da primeira RV e as mudanças ocorridas. 



\begin{tabular}{|l|l|}
\hline ASPECTOS INDENTIFICADOS & \multicolumn{1}{|c|}{ MUDANÇA OCORRIDA } \\
\hline Capacidade de dar e receber feedback & $\begin{array}{l}{[. . .] \text { na roda da vida, eu melhorei muito na capacidade de dar e }} \\
\text { receber feedback, isso aqui para mim foi muito bom [...]. (E8) }\end{array}$ \\
\hline Administração do tempo & $\begin{array}{l}\text { Minha roda na administração do tempo eu coloquei 30\%; eu posso } \\
\text { considerar hoje que melhorei, eu consigo aproveitar melhor o } \\
\text { tempo. (E7) }\end{array}$ \\
\hline Capacidade de lidar com pressão & $\begin{array}{l}\text { Aí, olhando aqui a Roda da Vida, eu coloquei a capacidade de lidar } \\
\text { com pressão; ela melhorou hoje porque, nas situaçós de pressão, } \\
\text { eu consigo fazer um pouco do que eu coloquei na meta ESPERTA. } \\
\text { (E5) }\end{array}$ \\
\hline $\begin{array}{l}\text { Comunicação } \\
\text { também estou aprendendo a me expressar. Ass vezes, eu explodia, } \\
\text { estou aprendendo a me controlar porque, às vezes, eu tenho razão, } \\
\text { mas, pela explosão, eu a perco. Saber falar no momento certo, calar } \\
\text { também no momento certo, estou aprendendo, isso é um processo. } \\
\text { (E7) }\end{array}$ \\
\hline $\begin{array}{l}\text { O papel da liderança, o curso foi muito bom. A gente aprendeu } \\
\text { que, para liderança, é importante o planejamento, aprender a } \\
\text { delegar, respeitar o momento das pessoas, apesar de não ser fácil } \\
\text { porque eu sou muito imediatista, porque eu não aguento e vou lá e } \\
\text { quero fazer sem esperar, isso eu estou controlando. (E7) }\end{array}$ \\
\hline
\end{tabular}

Quadro 3 - Descrição dos aspectos identificados como fragilidades e mudanças ocorridas (GOIÂNIA, 2014)

Para ilustrar alguns resultados da consolidação das mudanças ocorridas, os enfermeiros analisaram, por meio da $\mathrm{ME}$, os estados desejados para a mudança e os alcançados após seis meses da intervenção. Esse processo permitiu o reconhecimento e a descrição dos passos dados, ampliando a capacidade de os enfermeiros incorporarem suas mudanças desejadas. O quadro 4 ilustra o processo de alguns enfermeiros.

\begin{tabular}{|l|l|}
\hline \multicolumn{1}{|c|}{ ESTADO DESEJADO } & \multicolumn{1}{|c|}{ ESTADO ALCANÇADO } \\
\hline $\begin{array}{l}\text { Delegar responsabilidades e } \\
\text { orientar. }\end{array}$ & $\begin{array}{l}\text { A mudança mais importante foi a de aprender a delegar, era uma } \\
\text { dificuldade que eu tinha, me ajudou muito. (E6) }\end{array}$ \\
\hline $\begin{array}{l}\text { Ser equilibrada } \\
\text { emocionalmente. }\end{array}$ & $\begin{array}{l}\text { Eu acho que eu venho de um processo de mudança de amadurecimento, } \\
\text { principalmente, emocional porque, antes, eu era uma pessoa } \\
\text { extremamente imediatista. (E3) }\end{array}$ \\
\hline Lidar com conflitos. & $\begin{array}{l}\text { Hoje, eu consigo ter mais tranquilidade, coisa que antes que eu tinha } \\
\text { dificuldade, agora eu espero para despejar. (E8) }\end{array}$ \\
\hline Aprender a me comunicar. & $\begin{array}{l}\text { Eu aprendi muito a questão de como conversar, saber como conversar } \\
\text { com diferentes pessoas porque a gente conversa com diferentes pessoas } \\
\text { de diferentes estilos, e a saber esperar o tempo do outro. (E4) }\end{array}$ \\
\hline
\end{tabular}

Quadro 4 - Descrição do estado desejado e do estado alcançado (GOIÂNIA, 2014) 
Ferramentas do coaching no desenvolvimento da liderança da enfermagem na atenção... | 14

\section{Discussão}

O uso das ferramentas do coaching permitiu, aos enfermeiros, a percepção de suas dificuldades na liderança no contexto da APS. Deu-se visibilidade às suas fragilidades e apontaram-se caminhos para exercitar e consolidar mudanças, aprimorando suas competências na liderança.

A RV possibilitou, aos enfermeiros, identificar níveis baixos de satisfação em algumas áreas relacionadas à liderança. Assim, eles foram acionados a desenvolver autoconhecimento, o que, consequentemente, disparou o processo de mudança viabilizado pela conscientização de aspectos que exigiam mudanças. Estudos $2,14,16$ têm mostrado a importância do autoconhecimento no aprimoramento da capacidade para liderar. Uma pesquisa ${ }^{2}$ mostrou que os enfermeiros consideram que aprimorar seu autoconhecimento foi um benefício relevante adicional do processo de coaching.

Os resultados da pesquisa apontam a importância de se oportunizarem espaços que auxiliem os enfermeiros a reconhecerem suas competências gerenciais e suas limitações. O autoconhecimento é um caminho favorável para isso, o que permite, aos profissionais, melhorias no seu desempenho individual e organizacional. O aprimoramento dessas competências torna os enfermeiros mais empoderados para desempenharem suas funções e comprometidos com o fortalecimento e a implementação de reformas primordiais à APS. ${ }^{3-4,20}$

Em síntese, o autoconhecimento representa o primeiro passo a ser dado individualmente em direção à mudança. ${ }^{21} \mathrm{O}$ uso de ferramentas que favoreçam esse processo pode auxiliar os enfermeiros no desenvolvimento de sua liderança, permitindo que as pessoas reflitam a respeito de si mesmas, de sua visão de mundo, de seus valores e crenças. Assim, aprofundam sua aprendizagem e incorporam novas habilidades e novas capacidades. ${ }^{2,9,12}$

Ao analisar as metas estabelecidas pelos enfermeiros, constataram-se pontos de convergência em relação às necessidades definidas por eles para o aprimoramento. Entre os 
aspectos apontados, salienta-se a organização do tempo relacionada à priorização de tarefas e planejamento. Também foi comum a necessidade de melhorar a comunicação, especificamente no que diz respeito ao saber dar e receber feedback e na mediação de conflitos. A inteligência emocional e saber lidar com o estresse também foram apontados como metas prioritárias. Tais apontamentos deixam clara a necessidade do desenvolvimento de programas voltados para o aprimoramento da liderança, visto que esses quesitos são a base e o ponto de partida para uma boa liderança. ${ }^{7-9}$

Ao serem convidados a elaborar a $\mathrm{ME}$, os enfermeiros, mais conscientes de suas limitações em relação à liderança, elaboraram suas metas na busca por melhorias pessoais e para fortalecer o relacionamento dentro das equipes de trabalho. Desse modo, ficou clara a importância de melhorar a percepção do contexto de trabalho e das relações interpessoais, além da disponibilidade para o autoconhecimento permanente e o reconhecimento do outro dentro da equipe. Tais aspectos também foram reconhecidos como essenciais no aperfeiçoamento da liderança e apontados por enfermeiros submetidos ao processo de coaching em outras investigações. ${ }^{2,12,14,16}$ Estudo $^{13}$ destacou ainda que o coaching contribuiu para o desenvolvimento da liderança de enfermeiros que trabalham em práticas colaborativas. Esse resultado mostra o potencial do uso dessa ferramenta para enfermeiros que atuam na APS.

Nem sempre, o enfermeiro se vê preparado quando chamado a vivenciar um processo de coaching. ${ }^{2}$ Porém, o reconhecimento e a consciência de potencialidades e fragilidades mobilizaram esse profissional na busca por novos conhecimentos, o que fortalece o processo de mudança. Além disso, fica claro quando definem as evidências de como serão percebidas as mudanças. Os enfermeiros esperam que outros membros da equipe possam validá-los nesse processo, bem como o clima de trabalho seja modificado para melhor.

Esses resultados indicam o quanto o exercício da liderança do enfermeiro no contexto da APS pode ser valorizado com o melhor desempenho desses profissionais de forma mais assertiva. 
Ferramentas do coaching no desenvolvimento da liderança da enfermagem na atenção... | 16

A liderança é comprometida e fundamentada em conhecimentos da gestão, assim como indicam estudos realizados nessa perspectiva..$^{3-4,20,22-23}$

No que diz respeito aos dados relativos às mudanças apontadas pelos enfermeiros após seis meses de finalizado o programa de DL, pode-se observar que o uso das ferramentas do coaching proporcionou a identificação das carências e demandas, direcionou a mudança por meio de passos definidos e apresentou efeitos importantes com resultados positivos.

Entre o estado alcançado e o estado desejado pelos enfermeiros, percebeu-se que as ferramentas utilizadas durante o programa de DL proporcionaram melhoria no nível de satisfação das áreas apontadas na RV como prioritárias de desenvolvimento, especialmente a capacidade de dar e receber feedback, administração do tempo e comunicação. As principais mudanças sinalizadas pelos enfermeiros dizem respeito a aprender a delegar, ao amadurecimento emocional, à tranquilidade nas relações e a saber esperar o tempo do outro.

Na APS, é fundamental que o enfermeiro exerça uma liderança participativa que envolva toda a equipe. Estudos salientam a dificuldade que esses profissionais possuem em desenvolver esse papel. ${ }^{24-25}$ Nesse sentido, os resultados apontados pelos enfermeiros que participaram do estudo mostram como as ferramentas do coaching (RV e ME) foram essenciais para exercitarem suas capacidades de delegar, se sentirem mais tranquilos na função de líder, além do amadurecimento das relações.

O processo de mudança é visto, pelos enfermeiros, como necessário para a percepção de novos significados e novos hábitos. Assim, tomar consciência de si como sujeito do direcionamento das ações, tanto no trabalho quanto na vida pessoal, foi um dos ganhos importantes com essa experiência. 


\section{Considerações finais}

O modelo criado nesse programa de DL apresentou uma característica peculiar e inovadora, articulando ferramentas do coaching, permitindo unir experimentação e objetivação, levando os enfermeiros à transformação efetiva com a incorporação de mudanças no cotidiano do trabalho e na liderança da APS. Por meio dos resultados alcançados nesse programa de DL, tal como foi executado, é possível que ele seja reproduzido e utilizado não só para qualificar enfermeiros na APS, mas também para ser aplicado em outras áreas, nos diversos níveis de gestão.

O uso combinado das ferramentas RV e ME favoreceu o processo de mudança, fazendo o sujeito identificar a necessidade e tomar a decisão de mudar por direcionar a reflexão-ação rumo ao estado desejado. Dessa maneira, ressalta-se a importância do desenvolvimento de novos estudos de intervenção, ou seja, novos estudos que utilizem ferramentas do coaching e que o empreguem para promover mudanças não apenas na liderança, mas em outros aspectos da Enfermagem.

Para a Enfermagem, fica evidente a importância do uso de estratégias inovadoras no desenvolvimento da liderança de enfermeiros na APS, além de direcionar novos estudos que auxiliem a implementação desse tipo de ferramentas no contexto organizacional.

Considera-se, como limitações do estudo, a falta de pesquisas que descrevam o uso das ferramentas especificamente. Igualmente, o fato de nem todos os enfermeiros terem preenchido as ferramentas em questão, mesmo tendo participado sistematicamente do programa de DL. Esse, na verdade, é um risco calculado quando se utiliza pesquisa-ação. Além disso, os participantes comprometem-se, de modo único, cada um a avançar como pode nos processos de mudanças. 
Ferramentas do coaching no desenvolvimento da liderança da enfermagem na atenção... | 18

\section{Referências}

1. Guerrero-Núñez S, Cid-Henríquez P. Una reflexión sobre la autonomia y el liderazgo em enfermeria. Aquichan [Internet]. 2015 [acesso em 2017 abr 11];15(1):129-40. Disponível em: http://aquichan.unisabana.edu.co/index.php/aquichan/article/view/3511/3849

2. Westcott L. How coaching can play a key role in the development of nurse managers. J Clin Nurs [Internet]. 2016 [acesso em 2018 set 21];25(17-18):2669-77. Disponível em: https://www.ncbi.nlm.nih.gov/pubmed/27501254 doi: http://dx.doi.org/10.1111/jocn.13315

3. Lanzoni GMM, Meirelles BHS, Cummings G. Nurse leadership practices in primary healthcare: a grounded theory. Texto \& Contexto Enferm [Internet]. 2016 [acesso em 2018 maio 15];25(4):e4190015. Disponível em: http://www.scielo.br/pdf/tce/v25n4/pt_0104-0707-tce-25-04-4190015.pdf

4. Lowen IM, Peres AM, Crozeta K, Bernardinho E, Beck CLC. Managerial nursing competencies in the expansion of the Family Health Strategy. Rev Esc Enferm USP [Internet]. 2015 [acesso em 2018 maio 14];4996:967-73. Disponível em: http://www.scielo.br/pdf/reeusp/v49n6/pt_0080-6234-reeusp-49-060967.pdf

5. Munari DB, Bezerra ALQ, Nogueira AL, Rocha BS, Sousa ET, Ribeiro LCM. Leadership succession in nursing: thinking and acting today to ensure a better tomorrow. Rev Eletrônica Enferm [Internet]. 2017 [acesso em 2018 ago 21];19:1-5. Disponível em: https://revistas.ufg.br/fen/article/view/46101/22778

6. Hanse JJ, Harlin U, Jarebrant C, Ulin K, Winkel J. The impact of servant leadership dimensions on leade- member exchange among health care professionals. J Nurs Manag [Internet]. 2016 [acesso em 2018 maio 14];24(2):228-34. Disponível em: https://www.ncbi.nlm.nih.gov/pubmed/25879275

7. Mendes IAC, Ventura CAA, Trevizan MA, Marchi-Alves LM, Souza-Junior VD. Educação, liderança e parcerias: potencialidades da enfermagem para a cobertura universal de saúde. Rev Latinoam Enferm [Internet]. 2016 [acesso em 2018 abr 11];24:e2671. Disponível em: http://www.scielo.br/pdf/rlae/v24/pt_0104-1169-rlae-02673.pdf

8. Dyess SM, Sherman RO, Pratt BA, Chiang-Hanisko L. Growing nurse leaders: their perspectives on nursing leadership and today's practice environment. Online J Issues Nurs [Internet]. 2016 [acesso em 2018 jan 28];21(1):7. Disponível em: https://www.ncbi.nlm.nih.gov/pubmed/27853273

9. Cardoso MLAP, Ramos LH, D’innocenzo M. Liderança coaching: questionários de avaliação de percepções de líderes e liderados na enfermagem. Einstein [Internet]. 2014 [acesso em 2017 abr 10];12(1):66-74. Disponível em: http://www.scielo.br/pdf/eins/v12n1/pt_1679-4508-eins-12-1-0066.pdf 
10. Moore LW, Sublett C, Leahy C. Nurse managers' insights regarding their role highlight the need for practice changes. Appl Nurs Res [Internet]. 2016 [acesso em 2018 ago 15];30:98-103. Disponível em: https://www.ncbi.nlm.nih.gov/pubmed/27091262

11. Sociedade Brasileira de Programação Neurolinguística (SBPNL). Certificação Internacional em Coaching [Internet]. São Paulo; 2018 [acesso em 2019 junho 17]. Disponível em: https://www.pnl.com.br/certificacao-internacional-em-coaching/

12. Cable S, Graham E. "Leading Better Care": An evaluation of an accelerated coaching intervention for clinical nursing leadership development. J Nurs Manag [Internet]. 2018 [acesso em 2018 jul 14];26(5):60512. Disponível em: https://www.ncbi.nlm.nih.gov/pubmed/29600826

13. Graham R, Beuthin R. Exploring the effectiveness of multisource feedback and coaching with nurse praticioners. Nurs Leadership (Tor Ont) [Internet]. 2018 [acesso em 22 ago 2018];31(1):50-9. Disponível em: https://europepmc.org/abstract/med/29927383

14. Niesen CR, Kraft SJ, Meiers SJ. Use of motivational interviewing by nurse leaders: coaching for performance, professional development and career goal setting. Heath Care Manag [Internet]. 2018 [acesso em 22 ago 2018];37(2):183-92. Disponível em: https://www.ncbi.nlm.nih.gov/pubmed/29533243

15. Machado BP, Paes LG, Tonini TFF, Lampert NA, Lima SBS. Coaching en la enfermería: revisíon integradora. Index Enferm [Internet]. 2014 [acesso em 2017 abr 10];23(1-2):51-5. Disponível em: http://scielo.isciii.es/pdf/index/v23n1-2/revision1.pdf

16. Petty GM, Lingham T. Coaching team work in the classroom using an innovative team-coaching process. Nurs Educ Perspect [Internet]. 2018 [acesso em 2018 ago 19];40(2):118-20. Disponível em: https://www.ncbi.nlm.nih.gov/pubmed/29677042

17. Tripp, D. Pesquisa-ação: uma introdução metodológica. Educ Pesqui [Internet]. 2005 [acesso em 2017 abr 12];31(3):443-66. Disponível em: http://www.scielo.br/pdf/ep/v31n3/a09v31n3

18. Tong A, Sainsbury P, Craig J. Consolidated criteria for reporting qualitative research (COREQ): a 32item checklist for interviews and focus groups. Int J Qual Health Care [Internet]. 2007 [acesso em 2018 jul 11];19(6):349-57. Disponível em: https://www.ncbi.nlm.nih.gov/pubmed/17872937

19. Bardin, L. Análise de conteúdo. 7ª ed. São Paulo: Almedina Brasil; 2011.

20. Munyewende PO, Levin J, Rispel LC. An evaluation of the competencies of primary health care clinic nursing managers in two South African provinces. Glob Health Action [Internet]. 2016 [acesso em 2018 maio 14];9(1). Disponível em: https://www.tandfonline.com/doi/abs/10.3402/gha.v9.32486

21. Moscovici F. Desenvolvimento Interpessoal: treinamento em grupo. 17 ${ }^{\mathbf{a}}$ ed. Rio de Janeiro: José Olympio; 2009. 
Ferramentas do coaching no desenvolvimento da liderança da enfermagem na atenção... $\mid 20$

22. Lanzoni GMM, Meirelles BHS, Erdmann AL, Thofehrn MB, Dall'Agnol CM. Ações/intervenções motivadoras para liderança do enfermeiro no contexto da atenção básica à saúde. Texto \& Contexto Enferm [Internet]. 2015 [acesso em 2018 maio 15];24(4):1121-9. Disponível em: http://www.scielo.br/pdf/tce/2015nahead/pt_0104-0707-tce-201500003740013.pdf

23. Solà GJ, Badia JG, Delgado-Hito P, Osaba MAC, Del Val García JL. Self-perception of leadership styles and behaviour in primary health care. BMC Health Serv Res [Internet]. 2016 [acesso em 2018 maio 15];16(1):572. Disponível em: https://www.ncbi.nlm.nih.gov/pubmed/27733141

24. Carvalho AGF, Cunha ICKO, Balsanelli AP, Bernardes A. Liderança autêntica e perfil pessoal e profissional de enfermeiros. Acta Paul Enferm [Internet]. 2016 [acesso em 2018 maio 16];29(6):618-25. Disponível em: http://www.scielo.br/pdf/ape/v29n6/1982-0194-ape-29-06-0618.pdf

25. West M, Smithgall L, Rosler G, Winn E. Evaluation of a nurse leadership development programme. Nurs Manag (Harrow) [Internet]. 2016 [acesso em 2018 maio 16];22(10). Disponível em: https://www.ncbi.nlm.nih.gov/pubmed/26927790

\section{Autor correspondente}

E-mail: barbarasrocha@gmail.com

Endereço: Rua 1040 n.285 apt.502 Setor Pedro Ludovico

CEP: 74.823-250

\section{Contribuições de Autoria}

1 - Bárbara Souza Rocha

Concepção e planejamento do projeto de pesquisa, obtenção, análise e interpretação dos dados, redação e revisão crítica.

2 - Denize Bouttelet Munari

Concepção e planejamento do projeto de pesquisa, obtenção, análise e interpretação dos dados, redação e revisão crítica.

3 - Bruna Mendes de Sousa

Análise e interpretação dos dados, redação.

3 - Maria Lúcia Leal

Concepção e planejamento do projeto de pesquisa, obtenção, redação e revisão crítica.

4 - Luana Cássia Miranda Ribeiro

Redação e revisão crítica 
21 | Rocha BS, Munari DB, Sousa BM, Leal ML, Ribeiro LCM

\section{Como citar este artigo}

Rocha BS, Munari DB, Sousa BM, Leal ML, Ribeiro LCM. Ferramentas do coaching no desenvolvimento da liderança da enfermagem na atenção primária à saúde. Rev. Enferm. UFSM. 2019 [Acesso em: Anos Mês Dia];vol e46: P1-P23. DOI:https://doi.org/10.5902/2179769234762 